DOE Report No.

DE92 013588

\title{
Electronic States in Systems of Reduced Dimensionality
}

\author{
PROGRESS REPORT
}

for the period

September 15, 1991 - September 14, 1992

Grant Number: DE-FG02-87ER45334

Sergio E. Ulloa

Department of Physics and Astronomy and Condensed Matter and Surface Sciences Program

Ohio University, Athens, Ohio 45701-2979

Off.: (614) 593-1729

Fax: (614) 593-0433

ulloa@helios.phy.ohiou.edu

Date of subrnission: 15 April 1992

\section{DISCLAIMER}

\begin{abstract}
This report was prepared as an account of work sponsored by an agency of the United States Government. Neither the United States Government noi any agency thereof, nor any of their employees, makes any warranty, express or implied, or assumes any legal tiability or responsibility for the accuracy, completeness, or usefulness of any information, apparatus, product, or process disclosed, or represents that its use would not infringe privately owned rights. Reference herein to any specific commercial product, process, or service by trade name, trademark, manufacturer, or otherwise does not necessarily constitute or imply its endorsement, recommendation, or favoring by the United States Government or any agency thereof. The views and opinions of authors expressed herein do not necessarily state or reflect those of the United States Government or any agency thereof.
\end{abstract}

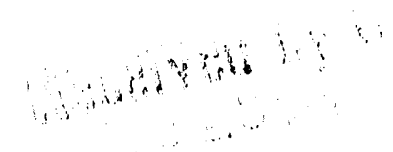




\section{Progress Report for the Year 1991-92}

The 91-92 granting period has been truly exciting for the project. We have completed problems proposed in our previous report, and continue working on some of the leads suggested by our results. We have also begun work in very promising new problems. All these efforts enhance our understanding of electronic systems with effectively-reduced dimensionality. In what follows we provide a summary of these efforts. Detailed descriptions are found in the technical reports attached as appendices to this report.

- Magnetically modulated systems. Together with Xiaoguang Wu (a postdoctoral fellow funded mostly by an Ohio University fellowship and partially by this grant), we have begun studies of the single-particle and collective excitations of novel two-dimensional electronic systems. In the proposed structures, the electrons move in the presence of moderately strong magnetic fields, so that only a few Landau levels are filled and/or important. However, the magnetic field is proposed to have an spatial strength modulation which has strong effects on the response of the system. In particular, we have analyzed the density-density correlation function (within a random phase approximation) for varying modulation strength and period. Increasing the strength of the modulation has the naturally expected result of forcing a crossover of the system into a one-dimensional regime. However, most interestingly, varying the period of the modulation for fixed magnetic field strengths also has strong crossover effects.

Reports of theoretical studies on these systems have only recently appeared, from us and others (the team of P. Vasilopoulus in Montreal and F. Peeters in Antwerp), and we intend to provide support for the possible experimental implementations of these systems. We are confident that typical structure sizes should give rise to strong mociulation effects. These devices are well within current state-of-the-art nanofabrication techniques, and preliminary conversations with experimental colleagues have been very encouraging.

A more technical discussion of these results is presented in two preprints attached to this report. One of them has been accepted for publication in Solid State Communications (DOE-ER45334-Pub\#13), and the other has been submitted to Physical Review (DOE-ER45334-Pub\#14).

- Inelastic magnetotunneling. Also in collaboration with Xiaoguang $\mathrm{Wu}$, we have studied the effects of phonon interaction in electronic magnetotunneling experiments in double-barrier multilayer systems. The observed strong Landau level 'replicas' 
produced by emission and absorption of phonons during the tunneling process are found to be in extremely good agreement with our theoretical calculations (see DOE-ER45334-Pub\#15 attached).

The electron-phonon interaction is assumed to be described by a Fröhlich term where the coupling matrix element is wave vector dependent. This allows for momentum exchange between electrons and phonons, and it is an important improvement over most earlier theoretical studies, where the phonon system is approximated by an independent boson model. The double-barrier tunneling system was studied using a transfer hamiltonian approach, which we believe should be appropriate for weak transmission. It is furthermore believed that this formulation possesses some advanlages over traditional wave function methods, especially when discussing scattering events in the tunneling process. It should also be applicable to other geometries, where lateral confinement is important. Further work along these lines is planned for the upcoming period (see following section).

- Ballistic transport review. During the period covered by this report, and together with Angus MacKinnon (Imperial College), Ele Castaño (UAM, Mexico) and George Kirczenow (Simon Fraser University), we have written a fairly substantial review entitled From Ballistic Transport to Localization, for the Volume I of the Handbook on Semiconductors, edited by P. T. Landsberg (University of Southampton), and to be published in October 1992 by Elsevier. This review is a substantial overview of ballistic transport and its overlap with localization studies and concepts. We are pleased with the results, and are getting good comments from copies distributed early. A copy is attached to this report (DOE-ER45334-Pub\#16).

- Screening in reduced dimensions. In collaboration with Dan Cheng (a student partially supported by this grant) we have also undertaken the study of electronelectron interactions and the competition with confinement, as manifested in the effectiveness of the electronic systems to screen external fields. We have been interested in the appearance of Friedel oscillations with multiple periods for the case of systems with reduced dimensions and more than one electronic subband occupied. These periods arise because of the segmented nature of the Fermi surface and the difference among the various characteristic lengths in reciprocal space. We have developed the formalism to describe the screening of various systems with reduced dimensionality (quasi-two-dimensional systems, quantum wires and quantum dots) and are currently studying the properties of different confinement potentials, subband occupation, and external magnetic fields. One of the most interesting results 
is the rather weak dependence of Friedel oscillations in quasi-one-dimensional systems, where the strongly reduced dimensionality yields a logarithmic decay of the screened interaction (within a random phase approximation). The relevance of these results to the well known properties of the non-Fermi-liquid nature of $1 \mathrm{D}$ systems is currently under study, and we expect to report on this soon. Preprints of this work will follow this report shortly.

- Raman and EELS in superlattices. Graduate student Xiaoju Wu (supported by the Condensed Matter and Surface Sciences program at Ohio University) has been working in the problem of calculating the dispersion relations for collective excitations in semiconductor superlattices. In particular, we have been looking at the effects of surface depletion present in realistic structures, and the signatures of the associated Tamm-like states. Raman probe yields and electron energy loss spectroscopy (EELS) in these structures have been chosen. These techniques appear to be the most naturally implementable tools to analyze the experimental behavior of both the single-particle and collective excitations in these systems. Unique dispersion relations have been uncovered using EELS-yield calculations, and have been reported at the recent March 1992 meeting of the American Physical Society. The theoretical formulation for the Raman scattering experiments is now complete and calculations are currently underway. We anticipate to be able to submit papers on these results soon. In the meantime, discussions with experimental colleagues at conferences have stirred some interest in the verification of our predictions. We are hopeful that these would be carried out in the near future.

A short invited review of some of the results in semiconductor superlattice systems has appeared in the magazine Visions, published by the Ohio Supercomputer Center, for dissemination in the supercomputer-user community in Ohio. A copy is attached (DOE-ER45334-Pub\#17).

- Ballistic quantum interference effects. Finally, together with graduate student Yong S. Joe (partially supported by this grant) we have been looking at the implementation of a novel configuration to observe the so-called electrostatic Aharonov-Bohm effect (EAB). The availability of ultraclean semiconductor devices where electrons can travel ballistically at low temperatures has opened a whole new area of research. We believe that the observation of the unique EAB is possible in these systems, as long as the suitable multiply-connected geometries can be implemented. The observation of the better known magnetic Aharonov-Bohm effect in these structures shows that this type of structures is feasible. Our preliminary 
results (reported at the March 1992 meeting of the American Physical Society in Indianapolis) are most encouraging. This development, in addition to touching on interesting fundamental properties of wave mechanics, should also yield novel applications for these structures, as discussed by us and others in the literature. We are very excited about the possibilities of such 'quantum interference' devices, and intend to carry out further work along these lines. Preprints describing results of this work are now being finished and will follow this project report soon.

We have tried to emphasize that our efforts on this project have yielded very interesting results on several camps. Experimental verification of some of our novel predictions and proposals should be forthcoming. We are very enthusiastic about ramifications and plan to pursue those accordingly, as described in the next section.

\section{Research Plans for the Year 1992-93}

Given the developments reported in the previous section, we plan to pursue extended studies of some of the points discussed, as well as to initiate other projects of current interest in the field. A brief description of some of our plans for this period follows:

- In the area of optical response of superlattices, we plan to formulate the problem of so-called type III and/or misaligned type II systems where electrons and holes coexist in either the same or in neighboring layers in doped structures. We anticipate that this problem will have a rich level structure and response functions, as a twocomponent fluid with charge carriers having opposite signs, as well as different masses. This structure should sustain electronic plasma modes, hole plasma modes, and possible mode mixing and anticrossing for near degenerate conditions. Since we have the theoretical 'machinery' to deal with this sort of interacting system, it should prove a relatively simple task. Our interest in this topic is now rekindled as recent experimental results by several groups in systems such as InAs-GaSb quantum wells have appeared, where our calculations could be tested.

- In the general area of ballistic transport in nanostructures, we plan to carry out additional studies, with emphasis on the properties observed under external magnetic fields, and transport through finite two-dimensional arrays of quantum 'dots' and 'antidots' properly coupled to carrier reservoirs. To this end, we plan to implement a fully tight-binding recursive Green's function algorithm with enough 
flexibility to incorporate different geometries and magnetic field strengths. Preliminary results indicate that a treatment in terms of traditional wavefunction expansions is not the most efficient approach in dealing with systems in strong magnetic fields. We believe that the recursive tight-binding approach should be better behaved. There is a great deal of interest on the experimental consequences of some of our results, as these systems represent some of the most interesting open problems in the field of ballistic transport.

- We will also be strongly involved in studying some of the problems arising in the novel magnetically modulated 2.D systems described in the previous section. A number of theoretical questions, such as the dependence of the various single-particle and collective excitations on the structural parameters will be studied. We are now analyzing the role of the relative ratio between the normalized modulation strength and the normalized period of the structure. We believe that especially for largeperiod systems, the appearance of novel edge states is possible, where these states will be localized around the regions where the field changes most rapidly. These states would have important effects on the transport properties of the system, sil h as the quantum Hall effect. We will also study the effects of two-dimensional modulation, instead of the one-dimensional modulation studied so far (see attachments), and the appearance of interesting crossover between a regular 2D and an array of quantum dots separated on two directions by the magnetic field modulation period. Possible self-consistent effects arising from electron-electron interactions will also be studied. We are very excited about the various possibilities with these novel structures.

- We have also begun efforts to estimate the effects of electrostatic potenuial modulation on two-dimensional excitons. Indeed, planar confinement of 2D excitons in heterojunctions and/or quantum wells is an area whose time has come. We believe that photoluminiscence (PL) and PLE should provide interesting new result: on the confinement of neutral excitations, giving rise to additional features with strength which is proportional to the amplitude of the confinement potentials. Fxperimentalists have expressed great interest in looking at these systems, and we are committed to provide theoretical guidance for the expected response. 


\section{Publications and Presentations for the Period}

Copies of the following reprints and preprints are attached to this report.

\section{A. Publications Prepared During Current Period}

1. X. Wu and S. E. Ulloa, Electronic levels and collective excitations in a two-dimensional system with a spatial magnetic field modulation, Solid State Commun. (1992), to appear [DOE-ER45334-Pub\#13].

2. X. Wu and S.E. Ulloa, Collective excitations in a magnetically-induced electronic dimensional crossover, submitted to Phys. Rev., October 1991 [DOE-ER45334Pub\#14].

3. X. Wu and S. E. Ulloa, Electron-phonon interaction in double-barrier resonant tunneling, Phys. Rev. B 44, 13148 (1991) [DOE-ER45334-Pub\#15].

4. S. E. Ulloa, A. MacKinnon, E. Castaño, and G. Kirczenow, From ballistic transport to localization, to appear in Handbook on Semiconductors, P.T. Landsberg, editor (North Holland, Amsterdam, 1992) [DOE-ER45334-Pub\#16].

5. S. E. Ulloa, Optical and Transport Properties of Electronic Structures, Visions (publication of the Ohio Supercomputer Center), Spring/Summer 1991, pp. 11-i5 [DOE-ER45334-Pub\#17].

\section{B. Reprints of Publications Previously Reported}

1. J. Zhan , S. E. Ulloa, and W. L. Schaich, Infrared active excitations in tunneling superlatt es and d-parameter theory, Phys. Rev. B 43, 9865 (1991) [DOE-ER45334Pub\#12].

\section{Presentations at Meetings and Seminars}

\section{A. Presentations by S. E. Ulloa}

- Contributed paper: D. Cheng, Y. S. Joe, and S. E. Ulloa, Screening and spectroscopy of impurities and obstacles in quantum wires, 9th International Conference on the Electronic Properties of Two-Dimensional Systems (EP2DS9), Nara, Japan, 8-12 July 1991. 
- Seminar: Quantum ballistic transport in electronic waveguides, Texas A\&M University, January 28, 1991.

- Seminar: Quantum ballistic transport in electronic waveguides, Ohio State University, 13 May 1991.

- Colloquium: Confined Electrons, First Colloquium on Physics of Surfaces, University of Sonora (CIFUS), Mexico, 23 May 1991.

- Seminar: Resonances, screening, and impurity scattering in nanoconstrictions, National Research Council, Ottawa, Canada, 2 September 1991.

- Colloquium: Optical and Transport Properties of Confined Electrons,

- Ball State University, IN, 24 October 1991.

- Wayne State University, MI, 20 November 1991.

- Contributed paper: N. Kim and S. E. Ulloa, Optical response of two-dimensional arrays of tunneling quantum dots, March Meeting of the American Physical Society, Indianapolis, 16-20 March 1992. Bull. Am. Phys. Soc. 37, 79 (1992).

\section{B. Presentations by Coauthors}

- Contributed paper: X. Wu and S. E. Ulloa, Electron-phonon interaction in double-barrier resonant tunneling, 38th Scottish Summer School in Physics of Nanosiructures, NATO ASI, St. Andrews, UK, 29 July - 9 August 1991. Presenter: Xiaoguang Wu.

- Contributed paper: X. Wu and S. E. Ulloa, Collective excitations of a twodimensional electron gas in a one-dimensional magnetic field modulation, 38th Scottish Summer School in Physics of Nanostructures, NATO ASI, St. Andrews, UK, 29 July - 9 August 1991. Presenter: Xiaoguang Wu.

- Contributed paper: Y.S. Joe and S. E. Ulloa, Electrostatic Aharonov-Bohm effect in quantum ballistic nanoconstrictions, 19th Midwest Solid State Theory Symposium, Michigan State University, October 6-7, 1991. Presenter: Y.S. Joe.

- Contributed paper: D. Cheng and S. E. Ulloa, Screening of ionized impurities in quasi-one- and two-dimensional electron systems, 19th Midwest Solid State Theory Symposium, Michigan State University, October 6-7, 1991. Presenter: D. Cheng. 
- Contributed paper: X. Wu and S. E. Ulloa, Collective excitations in a magnetically-induced electronic dimensional crossover, 19th Midwest Solid State Theory Symposium, Michigan State University, October 6-7, 1991. Presenter: Xiaoguang Wu.

- Contributed paper: Y. S. Joe and S. E. Ulloa, Electroconductance oscillations in quantum ballistic narrow channels, March Meeting of the American Physical Society, Indianapolis, 16-20 March 1992. Bull. Am. Phys. Soc. 37, 290 (1992). Presenter: Y.S. Joe.

- Contributed paper: X. Wu and S. E. Ulloa, Collective excitations in a magnetically-induced electronic dimensional crossover, March Meeting of the American Physical Society, Indianapolis, 16-20 March 1992. Bull. Am. Phys. Soc. 37, 533 (1992): Presenter: Xiaoguang Wu.

- Contributed paper: X. Wu and S. E. Ulloa, em Electron-electron scattering and collective excitations in doped semiconductor superlattices, March Meeting of the American Physical Society, Indianapolis, 16-20 March 1992. Bull. Am. Phys. Soc. 37, 606 (1992). Presenter: Xiaoju Wu.

- Contributed paper: D. Kachintsev and S. E. Ulloa, em CDW instability induced by magnetic field in planar electron systems, March Meeting of the American Physical Society, Indianapolis, 16-20 March 1992. Bull. Am. Phys. Soc. 37, 220 (1992). Presenter: D. Kachintsev.

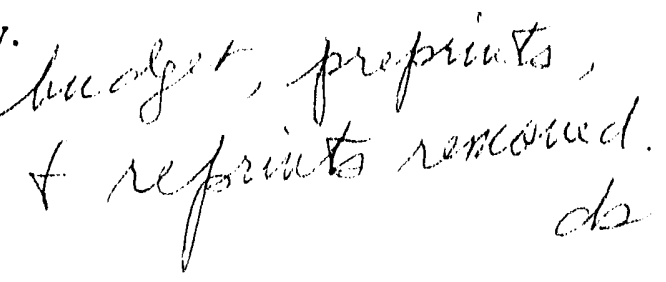



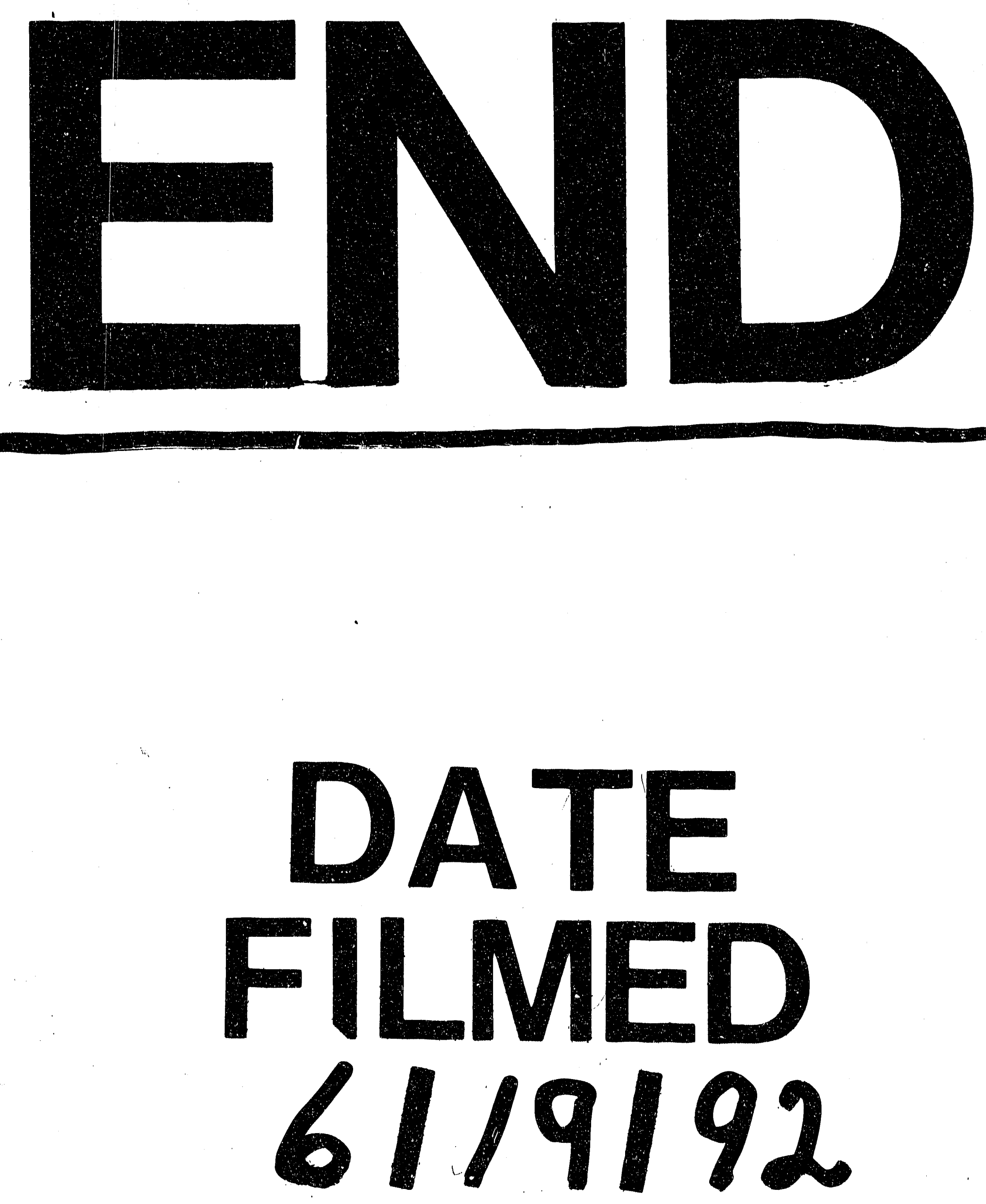
